\title{
Neurology
}

\section{Use of gold weights to correct lagophthalmos in neuromuscular disease}

Valeria Sansone, James Boynton and Cynthia Palenski

Neurology 1997;48;1500

DOI 10.1212/WNL.48.6.1500

This information is current as of November 4, 2012

The online version of this article, along with updated information and services, is located on the World Wide Web at:

http://www.neurology.org/content/48/6/1500

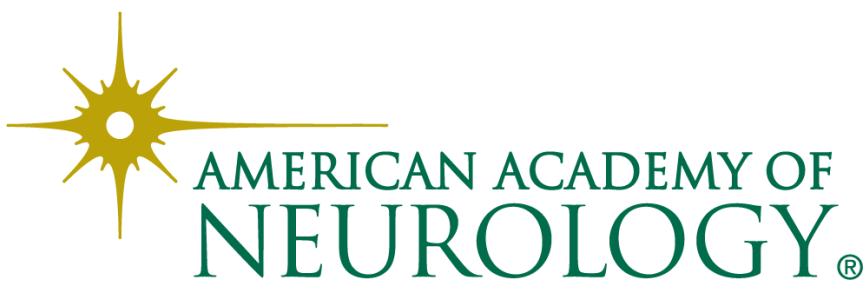




\title{
Use of gold weights to correct lagophthalmos in neuromuscular disease
}

\author{
Valeria Sansone, MD; James Boynton, MD; and Cynthia Palenski, MSNP
}

\begin{abstract}
Article abstract-Upper eyelid gold-weight implants are widely used in the correction of lagophthalmos in many neuromuscular conditions, most commonly facial palsy. The paralytic lagophthalmos that occurs in facioscapulohumeral muscular dystrophy (FSHD) is common and can cause severe ocular complications. It is not usually considered for surgical correction. Upper lid loading with $24 \mathrm{~K}$ gold implants and reconstructive lower lid surgery in a 64-year-old woman with FSHD corrected eyelid deformity and exposure keratitis. Surgical treatment also markedly improved facial appearance. This treatment may merit wider use in FSHD.
\end{abstract}

NEUROLOGY 1997;48:1500-1503

Paralytic lagophthalmos describes incomplete closure of the eyelids as a result of paralysis of the seventh cranial nerve, usually from lesions affecting the nuclear or peripheral portion of the nerve. Facial paralysis with resultant paralytic lagophthalmos and ectropion can occur from many causes, including Bell's palsy, tumors, trauma, injury, or vascular accidents affecting the facial nerve. ${ }^{1}$ There is significant variability in the onset and extent of facial nerve regeneration. The degree of recovery of facial nerve function after paralysis is influenced by the cause of the palsy, the degree of neural injury, the age of the patient, and the clinical setting. Whatever the cause, the ocular complications of inadequately or improperly managed facial paralysis range in severity from corneal irritation and punctuate keratopathy to corneal ulceration, perforation, and blindness.

Traditional medical therapy has included emollient ointments and eye drops, as well as taping and pressure patches. ${ }^{1,2}$ However, these are of shortlasting benefit and the frequency of application is such that these measures are often abandoned by the patients. This has led to use of surgical procedures in the management of facial paralysis. Partial or complete tarsorrhaphy has been the standard procedure of ophthalmologists for corneal protection. ${ }^{3}$ However, this does not improve active eyelid closure, it often restricts the visual field, and it is usually cosmetically displeasing to the patient. Such complications led to the development of a number of alternative surgical techniques to tarsorrhaphy.

One of the earliest approaches to repair upper lid ectropion due to paralytic lagophthalmos was the palpebral spring described by Morel-Fatio and Dalardrie, ${ }^{4}$ and was later modified. The spring, a stainless wire, is implanted in the lateral canthal area so that it forces the eyelid to close. When the eye is open, the levator palpebrae superioris muscle counteracts the force of the spring and holds the eyelids open. However, problems with implant extrusion and the need to adjust the tension of the spring have limited the acceptance of this procedure. Arion ${ }^{5}$ described a similar dynamic technique using a silicon rod placed in the pretarsal space of the upper and lower lids, encircling them and thus providing the force to close them. Extrusion and difficulty in adjusting the tension of the wire have also limited this technique's acceptance. Muhlbauer et al. ${ }^{6}$ placed small platinum magnets in the upper and lower eyelids, and used the magnetic force to close the eyelids. However, extrusion and infection were common. Other techniques such as temporalis muscle, facial nerve, and masseter muscle transfer ${ }^{7}$ have decreased the need for reoperation but are extremely timeconsuming and technically demanding.

Gold-weight implantation in the upper lid and surgery to tighten the lower eyelid are now routine procedures for the restoration of function and cosmesis to the paralyzed eyelids. First described by Sheehan, ${ }^{8}$ the lid-loading technique was modified by Smellie $^{9}$ by the use of gold as an implant material. This procedure was later popularized by $\mathrm{Jobe}^{10}$ and is now effectively and widely used in the treatment of facial palsy, from idiopathic nerve injury, trauma, tumors of the ear or of the brain, and from cerebrovascular accidents. ${ }^{2,11-15}$

Facioscapulohumeral muscular dystrophy (FSHD), the third most frequent form of muscular dystrophy, typically affects the muscles of the face, as well as the scapulae, upper arms, abdomen, and feet. ${ }^{16,17}$ The paralytic lagophthalmos that occurs in FSHD is common and can cause severe ocular complications. It is, however, not usually considered for surgical correction. Upper lid loading with $24 \mathrm{~K}$ gold implants and

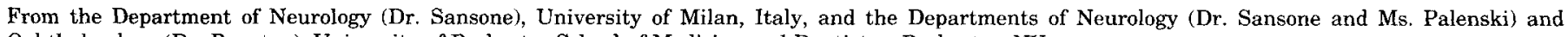
Ophthalmology (Dr. Boynton), University of Rochester School of Medicine and Dentistry, Rochester, NY.

This work was supported by NIH grant $220 \mathrm{G}$ and a grant from the MDA.

Received August 20, 1996. Accepted in final form December 26, 1996.

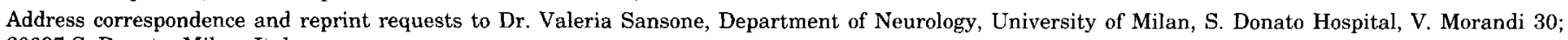
20097-S. Donato, Milan, Italy. 
reconstructive lid surgery in a 64-year-old woman with FSHD corrected eyelid deformity and exposure keratitis. Surgical treatment also markedly improved facial appearance. This treatment may merit wider use in FSHD.

Case history. A 64-year-old woman was evaluated for bilateral facial and proximal weakness. Since the age of 15 she had been unable to close her eyes completely, drink with a straw, or whistle. Pain and a feeling of 'sand in her eyes' were only temporarily relieved by the frequent application of eyedrops. Her past medical history was notable for hypertension and right parietal occipital intracerebral hemorrhage secondary to a vascular malformation. Neurologic examination showed prominent pseudobulbar release with emotional lability and mild cognitive impairment. $\mathrm{Bi}$ lateral facial and severe orbicularis weakness were present. Lid closure was greatly impaired and blinking was reduced. A 4-mm residual palpebral fissure was present on attempts at closure. Levator function appeared intact with good Bell's phenomenon. Lower lid movement was diminished with drooping and exposure of the lower portion of her corneas. Trichiasis caused irritation and tearing. Vision acuity was normal. Slit-lamp examination showed minimal drying inferiorly and bilateral superficial punctuate keratitis from incomplete lid closure and lash abrasion. Weakness of the orbicularis oris resulted in a typical transverse smile. Neck flexors were normal. Scapular winging and upper arm weakness were present and more evident on the left side. In the lower limbs there was mild to moderate bilateral proximal weakness, more evident on the left side, associated with atrophy of the thigh muscles. Beevor's sign was absent. Reflexes were brisk on the left, there was a left Babinski sign, and sensation was slightly reduced to pinprick on the left. Romberg was positive. Functionally the patient could not get up from a squat, but could get out of a chair unaided. Gait was broad based and waddling. Her 34-year-old son had a similar distribution of muscle weakness. Muscle biopsy and EMG were consistent with a chronic myopathy.

Surgical procedure. Reconstructive surgery was carried out to reduce corneal exposure, improve the effectiveness of blinking, and eliminate the marginal entropion. Identical procedures were performed on both eyelids using local anesthesia.

A $24 \mathrm{~K}$ gold weight (0.6 g MedDev Corporation [Los Altos, CA]) was placed in a pretarsal pocket in the upper lid and sutured to the tarsus. ${ }^{10-14,18}$ The paralytic ectropion was repaired by tightening the lateral canthal tendon. ${ }^{2,3}$ Catgut sutures were passed through the full thickness of the lower lid to correct the marginal entropion. ${ }^{2} \mathrm{~A}$ medial canthoplasty raised the medial aspect of the lower lid to reduce exposure. ${ }^{18}$

Results. Postoperatively the patient experienced a marked improvement in ocular comfort and better vision subjectively. There was improvement of blinking and closure of the eyelids. In the closed eyelid position, the palpebral fissure opening was $4 \mathrm{~mm}$ bilaterally preoperatively (figures A and B). Complete closure was obtained after surgery bilaterally (figure D). Nocturnal exposure was no longer present. Mild ptosis (3 $\mathrm{mm}$ below the upper corneal limbus) was noted 2 weeks after the surgery bilaterally (figure C). There was, however, no restriction of superior visual field, and the patient was pleased with the lid position. There was no rejection, exposure, or infection of the weights and surrounding tissues after 1 year of follow-up.

Discussion. Tarsorraphy has been the usual palliative treatment of facial paralysis. ${ }^{3}$ However, it has many limitations: it does not improve active eyelid closure, it restricts peripheral vision, and it is cosmetically displeasing. ${ }^{2}$ Various prosthetic devices like wire springs, ${ }^{419}$ a tantalum gauze mesh, ${ }^{8}$ magnets, ${ }^{6}$ and rubber band strips, ${ }^{5}$ have been used to improve lagophthalmos. These techniques often require surgical revision, and have been associated with infection and extrusion.

Weighting of the upper lid with gold to provide closure and cosmetic improvement in lagophthalmos and keratopathy due to facial palsy has found increasing acceptance. ${ }^{10,12-14,18}$ It has been used to treat facial paralyses resulting from trauma, tumors, infections, and vascular malformations, with few adverse side effects. ${ }^{10}$ This report documents successful gold-weight implantation to treat lagophthalmos and exposure keratopathy in a patient with FSHD. Such patients are seldom considered for surgical treatment. We have not had the opportunity to perform any kinematic studies to document the clinical impressions of improvement in this patient. ${ }^{15}$ Although these studies are available only at a limited number of centers, a quantitative evaluation of blink kinematics is important to assess improvement and for follow-up evaluation of recovery of blink function.

Gold weights have several advantages: (1) they are maintenance free, (2) implantation can be performed under local anesthesia, (3) inflammatory reactions are uncommon, ${ }^{20}(4)$ the procedure is reversible without leaving any defects, (5) implantation can be combined with other surgical techniques (such as a lower lid tightening procedure) to obtain maximum corneal protection (marginal entropion, lower lid ectropion repair, and medial canthoplasty were performed concomitantly in our patient, (6) ocular cosmesis is successfully obtained, and (7) gold is an inert metal that allows MRI scans if necessary.

Ptosis can occur from the weight, but the levator muscle of the upper eyelid tends to overcome the initial droop after about 6 weeks. The weight does not interfere with elevation of the eyelid, but allows the lid to fall when the levator relaxes, as in a downward gaze, blinking, and lid closure. Elevation of the head at night may be helpful after lid loading to ensure complete corneal protection at night. This precaution may not be necessary when eyelid loading is combined with other procedures like medial canthoplasty. Implantation in our patient resulted in a mild degree of ptosis that, however, did not interfere with field vision and was well tolerated by the patient. Extrusion of the implant in previous reports ${ }^{20}$ occurred 3 to 4 months postoperatively in a small number of patients.

Upper lid loading with a gold implant is a rela- 


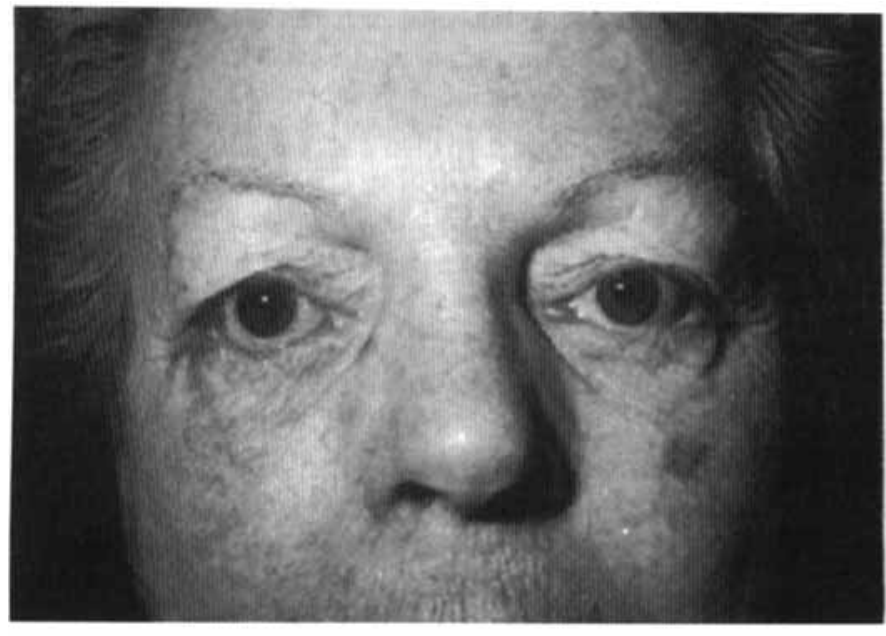

A

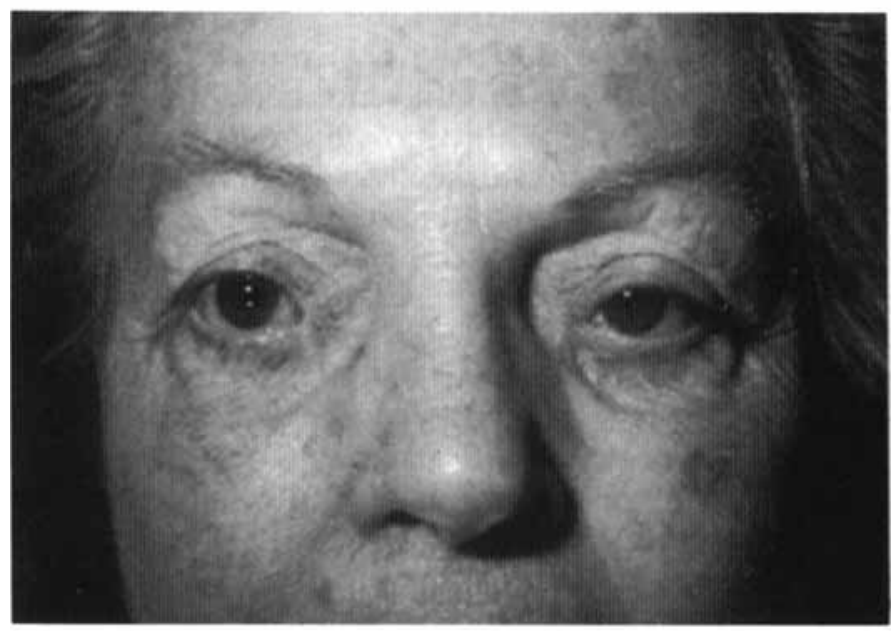

C

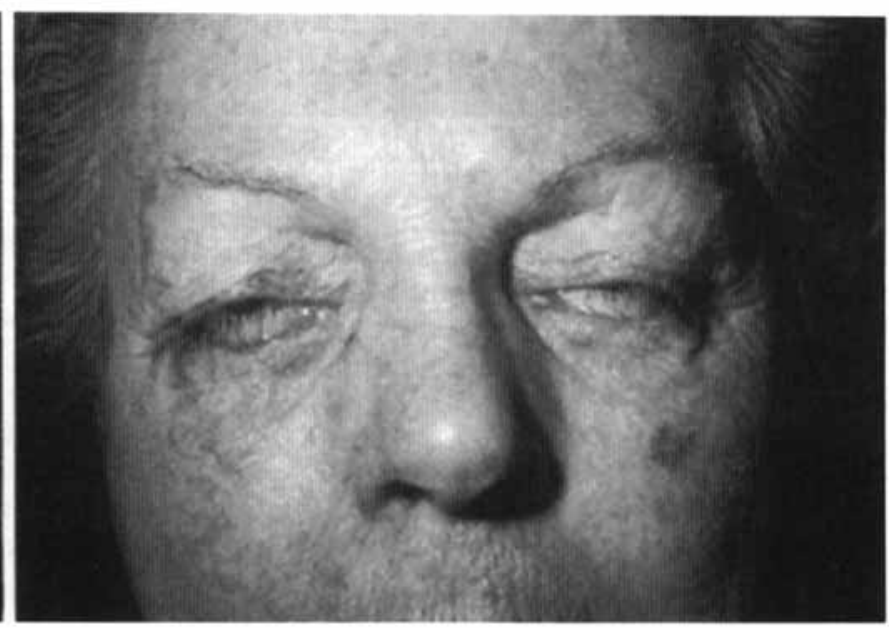

B

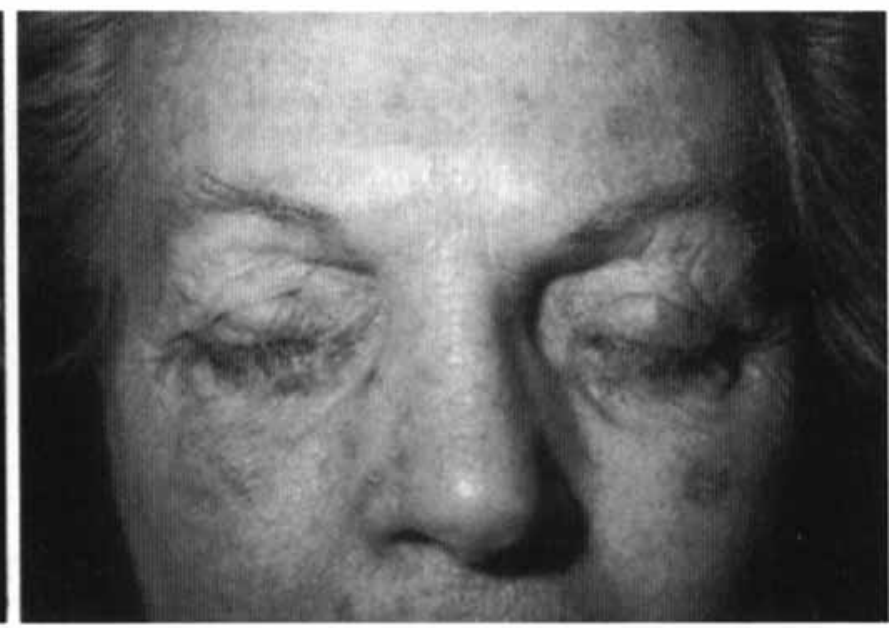

D

Figure. (A, B) Preoperative photographs of patient with facioscapulohumeral muscular dystrophy. (C, D) Postoperative photographs 12 months after bilateral gold-weight upper eyelid implantation, radial canthoplasty, and repair of lower lid marginal entropion. Complete eyelid closure was obtained on relaxation of the levator muscle.

tively simple procedure to perform, produces acceptable functional and cosmetic improvement, and has a relatively low complication rate. It should be considered as an early therapeutic measure to relieve discomfort and to prevent severe keratopathy resulting from excessive corneal exposure in FSHD and other neuromuscular diseases causing lagophthalmos. The multidisciplinary approach to the care of these patients is strongly encouraged.

\section{Acknowledgment}

The authors wish to thank Lisa Oppelt for help with preparation of the manuscript.

\section{References}

1. Levine RE, May M. Ophthalmologic medical management. In: May M, ed. The facial nerve. New York: Thieme Medical, 1986:339-352.

2. May M. Gold weight and wire springs implants as alternatives to tarsorraphy. Arch Otolaryngol Head Neck Surg 1987;113: 656-660.

3. Mausolg FA. Techniques for the repair of orbicularis oculi palsy. Ophthalmic Surg 1978;9:67-70.

4. Morel-Fatio D, Dalardrie JP. Palliative surgical treatment of facial paralysis. The palpebral spring. Plast Reconstr Surg 1964;33:446- 456 .

5. Arion HG. Dynamic closure of the lids in paralysis of the orbicularis muscle. Int Surg 1972;57:48-51.

6. Muhlbauer WD, Segeth H, Viessmann A. Restoration of lid function in facial palsy with permanent magnets. Chir Maxillofac Plast 1973;1:295-304.

7. Baker DC, Conley J. Regional muscle transposition for rehabilitation of the paralyzed face. Clin Plast Surg 1979;6:317331.

8. Sheehan JE. Progress in correction of facial palsy with tantalum wire and mesh. Surgery 1950;27:122-125.

9. Smellie GD. Restoration of the blinking reflex in facial palsy by a simple lid-loading operation. Br J Plast Surg 1966;19: $279-283$.

10. Jobe RP. A technique for lid loading in the management of the lagophthalmos of facial palsy. Plast Reconstr Surg 1974;53: $29-32$.

11. Chapman MP, Lamberty BGH. Results of upper lid loading in 
the treatment of lagophthalmos caused by facial palsy. $\mathrm{Br} \mathrm{J}$ Plast Surg 1988;41:369-372.

12. Gilbard SM, Daspit CP. Reanimation of the paretic eyelid using gold weight implantation. A new approach and prospective evaluation. Ophthal Plast Reconstr Surg 1991;7:93-103.

13. Gladstone GJ, Nesi FA. Management of paralytic lagophthalmos with a modified gold-weight implantation technique. Ophthal Plast Reconstr Surg 1996;12:38-44.

14. Kartush JM, Linstrom CJ, McCann PM, et al. Early gold weight eyelid implantation for facial paralysis. Otolaryngol Head Neck Surg 1990;103:1016-1023.

15. Chuke JC, Baker RS, Porter JD. Bell's palsy: associated blepharospasm relieved by aiding eyelid closure. Ann Neurol 1996;39:263-268.

16. Tawil R, Forrester J, Griggs RC, et al. Evidence for anticipa- tion and association of deletion size with severity in facioscapulohumeral muscular dystrophy. Ann Neurol 1996;39: 744-748.

17. Tawil R, Griggs RC. Facioscapulohumeral muscular dystrophy. In: Rosenberg RN, Prusiner SB, DiMauro S, Barchi RL, eds. The molecular and genetic basis of neurologic disease. Newton, MA: Butterworth Heinemann, 1996.

18. Custer PL, Tenzel RR. Lateral canthal sling update. Ophthal Plast Reconstr Surg 1985;1:121-127.

19. McNeill JI, Oh Y. An improved palpebral spring for the management of paralytic lagophthalmos. Ophthalmology 1991;98: $715-719$.

20. Bair RL, Harris GJ, Lyon DB, et al. Noninfectious inflammatory response to gold weight eyelid implants. Ophthal Plast Reconstr Surg 1995;11:209-214. 
Use of gold weights to correct lagophthalmos in neuromuscular disease

Valeria Sansone, James Boynton and Cynthia Palenski

Neurology 1997;48;1500

DOI 10.1212/WNL.48.6.1500

This information is current as of November 4, 2012

Updated Information \& Services

Permissions \& Licensing

Reprints including high resolution figures, can be found at: http://www.neurology.org/content/48/6/1500

Information about reproducing this article in parts (figures, tables) or in its entirety can be found online at:

http://www.neurology.org/misc/about.xhtml\#permissions

Information about ordering reprints can be found online: http://www.neurology.org/misc/addir.xhtml\#reprintsus 